DRAFT VERSION DECEMBER 5, 2018

Preprint typeset using LTEX style emulateapj v. 08/22/09

\title{
INTRACLUSTER SHORT GAMMA-RAY BURSTS BY COMPACT BINARY MERGERS
}

\author{
YUU NIINO ${ }^{1,2}$ AND TOMONORI TOTANI ${ }^{1}$ \\ Draft version December 5, 2018
}

\begin{abstract}
One of the possible origins of short gamma-ray bursts (SGRBs) is merging of compact binaries, and the effect of large kick velocity is a signature that can be used as an observational test for this hypothesis. Intracluster SGRBs that escaped from a host galaxy in a galaxy cluster are interesting in this context, since they would escape more easily by cluster tidal force, and would have brighter afterglow luminosity by dense intracluster gas, than those in general field galaxies. Here we calculate the escape fraction of compact binaries from their host galaxies in a galaxy cluster, and discuss some observational implications. We found that the escape fraction strongly depends on the nature of dark matter subhalos associated with member galaxies. If the amount of dark matter around member galaxies is not large and the gravitational potential for an escaping binary is determined mostly by stellar mass, most of SGRBs should escape and be observed as hostless, which is a much higher fraction than those in the field. Hence, statistics of intracluster SGRBs could give important information about the dark matter distribution in galaxy clusters, as well as hints for the origin of SGRBs.
\end{abstract}

Subject headings: binaries: general — gamma rays: bursts — galaxies: clusters: general

\section{INTRODUCTION}

Short gamma-ray bursts (SGRBs) occur not only in star forming galaxies but also in early type galaxies, indicating a longer time scale of the delay from star formation to the SGRB events (Gehrels et al. 2005; Villasenor et al. 2005; Berger et al. 2005) than that of the long gamma-ray bursts (LGRBs) that are associated with massive star collapses [see Piran (2004); Nakar (2007) and Lee \& Ramirez-Ruiz (2007) for reviews]. A strong candidate for the origin of SGRBs satisfying this nature is merging of compact object binaries (e.g., double neutron star binaries or neutron star black hole binaries).

Compact binaries are expected to have large systemic velocities by the kick given at the time of compact object formation, as inferred from the large proper motion of pulsars. Because of the long time scale and the large velocity, SGRBs are expected to occur with a large offset from the centers of galaxies compared with stellar distribution. There are some model calculations of this effect (Bulik, Belczyński, \& Zbijewski 1999; Bloom, Sigurdsson, \& Pols 1999; Fryer, Woosley,\& Hartmann 1999) and such an expectation is indeed consistent with observations, though the statistics is still limited (Lee et al. 2005; Fox et al. 2005; Bloom et al. 2007). However, truly intergalactic SGRBs that are ejected from their host galaxies would be difficult to identify, because of the difficulty to identify their host galaxies. Furthermore, we do not expect a strong afterglow emission in low density environment in the intergalactic field, making the identification even more difficult [see also Bloom et al. (2006); Nakar (2007)].

SGRBs in galaxy clusters are particularly interesting in this context, since compact binaries would be more efficiently stripped away from their host galaxies and become intracluster SGRBs, because of the strong tidal force in the cluster gravity field. The idea of tidal stripping is consistent with observations of intracluster stars (Vílchez-Gómez et al. 1994; Okamura et al. 2002; Durrell et al. 2002; Gal-Yam et al.

\footnotetext{
${ }^{1}$ Department of Astronomy, School of Science, Kyoto University, Sakyoku, Kyoto 606-8502, Japan

2 niinou@kusastro.kyoto-u.ac.jp
}

2003; Gerhard et al. 2007). We expect that such intracluster SGRBs could have brighter afterglows because of the denser environment of the intracluster gas than that in the intergalactic field, which would help the accurate determination of SGRB locations. A few SGRBs have already been observed in galaxy clusters, though the sample is still small (Berger et al. 2007; Shin \& Berger 2007). The statistics of such intracluster SGRBs in the future data would then give us some information about the origin of SGRBs, and the gravitational potential of galaxies in a cluster.

Here we calculate the expected event rate of such intracluster SGRBs, assuming that SGRBs are indeed the products of compact binary mergers. We also discuss possible implications for future observations. We assume $\Lambda$ CDM cosmology with $\Omega_{\Lambda}=0.7, \Omega_{M}=0.3$, and $H_{0}=70 \mathrm{~km} \mathrm{~s}^{-1} \mathrm{Mpc}^{-1}$.

\section{FORMULATIONS}

\subsection{Models of a Galaxy Cluster and Its Member Galaxies at $z=0$}

First we construct a model of a galaxy cluster at $z=0$, and will consider its evolution in the next subsection. As a typical galaxy cluster, we consider a spherically symmetric cluster with a total cluster mass of $10^{15} M_{\odot}$, a virial radius of $R_{\mathrm{vir}}=1 \mathrm{Mpc}$, and the Navarro-Frenk-White (NFW, Navarro, Frenk, \& White 1995) density profile for dark matter (DM) with a concentration parameter of $c_{\text {halo }}=5$. All these parameters are typical for a rich cluster [e.g., Schmidt \& Allen (2006)]. We assume the Schechter function for the luminosity function of member galaxies, with the shape parameters (i.e., $L_{*}$ and $\alpha$ ) independent of radius from the cluster center, $R$. We use the estimate of the Schechter parameters by the Sloan Digital Sky Survey data for galaxy clusters (Hansen et al. 2005, Table 2). The $R$-dependence of the normalization of the luminosity function, $\phi_{*}$, is assumed to be the NFW profile, but with the concentration parameter $c_{\text {galaxv }}=1.2$ that is different from that for the dark matter (Hansen et al. 2005).

We then determine the stellar mass and density profile within a member galaxy as follows. It is well known that early-type galaxies are the dominant population in rich galaxy clusters (Oemler 1974; Thomas \& Katgerd 2006), and we 
assume all cluster galaxies to be elliptical galaxies for the simplicity. The observed luminosity function is in the $r^{\prime}$ band, and we translate it into the $B$ band by a typical color of elliptical galaxies, $B-r^{\prime}=-1.4$. We assume the ratio of the stellar mass to light to be $9.8 M_{\odot} / L_{\odot}$ in the $B$ band. These quantities are from the model used by Totani \& Yoshii (2000), which can reproduce the observed galaxy properties well. Stellar density profile within member galaxies is assumed to be the Hernquist profile (Hernquist 1990), with the characteristic radius parameter chosen so that a half of the total mass is included within the observed half-light radius, $r_{\text {half }}$, which is calculated by the power-law relation to $B$ luminosity fitted to observations (Totani \& Yoshii 2000).

On the other hand, the density profile of dark matter associated with the cluster member galaxies as substructure in the whole cluster is uncertain and poorly known. Especially, the dark matter subhalos extending to the outer region of member galaxies are expected to be vulnerable to the tidal forces by galaxy interactions and/or the overall cluster gravitational potential. Here we consider the two extreme cases: (i) there is no significant DM substructure or subhalos associated with member galaxies, and the gravitational potential well of galaxies is determined simply by the stellar mass profile (the no subhalo case), and (ii) the DM subhalos are associated with member galaxies with a similar amount to the field galaxies (the preserved subhalo case). The amount and nature of subhalos depend on the cluster forming processes, and probably the reality is between these two extreme cases.

For the preserved subhalo case, we calculate the virial mass $\left(M_{\mathrm{vir}}\right)$ of the subhalos from the stellar mass of a galaxy and the universal ratio of the dark-to-baryonic matter $\Omega_{M} / \Omega_{b}=$ 5.9 (Spergel et al. 2003). The virial radius $\left(r_{\text {vir }}\right)$ of the subhalo is calculated from $M_{\text {vir }}$ and the 1-dimensional central velocity dispersion $\left(\sigma_{v}\right)$ of the galaxy, as $G M_{\mathrm{vir}} / 2 r_{\mathrm{vir}}=$ $3 \sigma_{v}^{2}$. Here, the velocity dispersion is that for stars calculated from the galaxy luminosity using the Faber-Jackson relation (de Vaucouleurs \& Olson 1982). An almost similar velocity dispersion is obtained also from the stellar mass and $r_{\text {half }}$ calculated above and assuming the virial relation, giving a consistency check for our treatment. We assume the NFW profile for the DM subhalos, and the concentration parameter is calculated by the formula given by Bullock et al. (2001) for subhalos included in larger virialized halos, which are based on cosmological N-body simulations: $c \simeq c_{*}\left(M_{\mathrm{vir}} / M_{*}\right)^{\Gamma}$ at $z=0$ where $c_{*}=7, \Gamma=-0.3$, and $M_{*} \simeq 1.5 \times 10^{13} h^{-1} M_{\odot}$.

\subsection{Cluster and Galaxy Evolution}

It is well known that most stars in elliptical galaxies formed at high redshift $(z>1)$ and they evolved passively to the present time (e.g., Yamada et al. 2005). Major mergers could change drastically the stellar mass distribution and gravitational potential of member galaxies, but a recent numerical simulations by Murante et al. (2007) indicates that the majority of member galaxies in a cluster do not undergo major mergers, except for the brightest central galaxy which is formed in the collision of many galaxies. Therefore we make a reasonable assumption that the stellar mass distribution does not evolve in cluster member galaxies.

Though galaxies form at the early epoch of $z>1$, the establishment of the overall cluster potential should be significantly later according to the standard picture of hierarchical structure formation. We estimate the epoch of cluster formation using the extended Press-Schechter approximation (Lacey \& Cole
1993). It predicts that about half of mass of a $10^{15} M_{\odot}$ cluster at $z=0$ is already included in the largest progenitor at $z_{F} \sim 0.45$. Therefore we assume that the tidal force by the cluster potential starts to affect member galaxies at this redshift. We do not take into account the evolution of the cluster potential at $z<z_{F}$, and this is a reasonable approximation because the time scale of cluster potential evolution is much larger than the orbital period of compact binaries in member galaxies. The evolution of DM subhalos is difficult to predict without detailed numerical simulations, and we simply apply the above two extreme models with no evolution, which would cover the realistic evolution.

\subsection{Escape of Compact Object Binaries}

In a galaxy with a given luminosity, we can calculate the orbit of compact object binaries in the gravitational potential as modeled in $\$ 2.1$ if the initial location and velocity are given. We calculate the initial velocity by the sum of the original stellar velocity $\mathbf{v}_{\text {org }}$ at the location and the kick velocity $\mathbf{v}_{\text {kick }}$ given when the compact objects are formed. The Maxwell distribution having the 1-dimensional velocity dispersion $\sigma_{v}$ defined in the previous section is assumed for $\mathbf{v}_{\text {org }}$. The direction of both $\mathbf{v}_{\text {org }}$ and $\mathbf{v}_{\text {kick }}$ are assumed to be isotropic and random.

Although the velocity distribution of observed single pulsars is well fitted by a Gaussian (Hobbs et al. 2005), that for compact object binaries is not well known. A few observed binary pulsars have bulk motion velocities of $\sim 100-200$ km/s (Wex, Kalogera, \& Kramer 2000; Ransom et al. 2004; Willems, Kalogera, \& Henninger 2004). Bulik et al. (1999), Bloom et al. (1999) and Fryer et al. (1999) theoretically estimated the velocities of compact binaries that remain gravitationally bound after supernova explosions, to be several hundreds $\mathrm{km} / \mathrm{s}$. We assume the distribution of $\mathbf{v}_{\text {kick }}$ to be a single isotropic Gaussian, i.e., each 1-dimensional component of $\mathbf{v}_{\text {kick }}$ is a Gaussian with the standard deviation $\sigma_{k}$. We calculate the cases of two different values of $\sigma_{k}=100$ and 300 $\mathrm{km} / \mathrm{s}$. The mean (the standard deviation) of the corresponding $\left|\mathbf{v}_{\text {kick }}\right|$ distribution then becomes 160 (66) and 480 (200) km/s for $\sigma_{k}=100$ and $300 \mathrm{~km} / \mathrm{s}$, respectively.

We solve the motion of binaries until their merger time (the time from a compact binary formation to its merger by gravitational wave radiation). Though the merger time generally ranges more than three orders of magnitude, $10^{7-10} \mathrm{yr}$ (e.g., Tutukov \& Yungelson 1994; Bulik et al. 1999), we are interested in SGRBs in galaxy clusters. Most of galaxies in clusters have formed their stars at high redshift $(z \gtrsim 2)$, and observed SGRBs are typically at $z \sim 0.2$. Therefore, the time between these epochs, i.e., $\sim 10^{10}$ is appropriate for the merger time in this work.

We consider that a binary has escaped from its host galaxy once its distance from the host galaxy center becomes larger than the tidal radius $r_{\text {tide }}$ of the host galaxy after the formation of the galaxy cluster, i.e., $z<z_{F}$. Here, the tidal radius is defined as the radius where the tidal force by the overall cluster potential is the same as the binding force in the host galaxy. The tidal radius is numerically calculated for a given set of galaxy luminosity and location in the cluster. We then calculate the mean escape fraction as a function of $R$, taking a weighted average over the host galaxy luminosity, initial location in the host, and kick velocity.

It should be noted that some fraction of stars are distributed at $r>r_{\text {tide }}$ with the assumed stellar mass profile in host galax- 
ies. Such stars would be stripped from host galaxies and become intracluster stars. Compact object binaries in such stellar populations would all contribute to the intracluster SGRBs. We find that this fraction is about $2 \%$ in the preserved subhalo case and $10 \%$ in the no subhalo case. It seems that the no subhalo case is preferred (see 4.2), from a comparison of these values with the various observational estimates of the abundance of intracluster stars in galaxy clusters.

\section{RESULTS}

The results are shown in Figure 1, where the mean escape fraction within a given radius from the cluster center, $f_{\text {esc }}(<R)$, is plotted. We find that the escape fraction largely depends on the existence of the DM substructure; the escape fraction is modest with $f_{\text {esc }} \sim 0.2$ in the preserved subhalo case, while most binaries will be ejected in the no subhalo case. The dependence on the radius from the cluster center or on the kick velocity is not as significant as the effect of subhalos within the parameter ranges investigated.

For comparison, we calculate the case of field galaxies not in clusters. Again, we only consider elliptical galaxies, and their properties are calculated in the same way for a given luminosity. Bullock et al. (2001) found that isolated halos have a different relation between the virial mass and the concentration parameter from that for subhalos, and we adopt $c_{*}=9$ and $\Gamma=-0.13$ here based on their results. The escape fraction averaged over galaxy luminosity is simply calculated without taking into account the tidal force by external gravity field. Here we use the luminosity function shape parameters of Blanton et al. (2001) for field galaxies; though these are derived for all types of field galaxies, the luminosity function for each galaxy type is rather uncertain. The results are shown in Table 1, and we found that the escape fraction in the field is not much different from that in galaxy clusters in the preserved subhalo case, while a large enhancement of the cluster $f_{\text {esc }}$ is predicted in the case of no subhalos.

\section{DISCUSSION}

\subsection{Detectability of SGRB Afterglows in Clusters}

Detection of afterglows is necessary to locate a GRB accurately enough with respect to a host galaxy. We discuss here the detectability of a typical SGRB afterglow in the intracluster medium following the standard afterglow model of Sari, Piran, \& Narayan (1998). We simply use this isotropic model without jet structure by using the isotropic equivalent total energy. The jet break may reduce the expected flux at a later time compared with the calculation here, but this crude estimate is sufficient here for our purpose.

The isotropic-equivalent total energy in gamma-rays of SGRBs is distributed in a wide range of $E_{\gamma, \text { iso }} \sim 10^{49}$ $10^{51} \mathrm{erg}$, and the total initial kinetic energy of the external shock $\left(E_{\text {iso }}\right)$ is expected to be similar (Fox et al. 2005; Soderberg et al. 2006). According to Panaitescu \& Kumar (2001), we adopt the following parameters: the fraction of energy density in magnetic field $\epsilon_{B}=10^{-2.4}$ and that in nonthermal electrons $\epsilon_{e}=10^{-1.2}$; although these are for LGRBs, the values inferred for available SGRBs are not much different. We also assume the power index of the luminosity decay, $\alpha=-1$ where $F_{\nu} \propto t^{\alpha}$. The typical particle density of intracluster medium is $n \sim 10^{-3} \mathrm{~cm}^{-3}$ within a few hundreds kpc from the cluster center (e.g., Lewis, Buote, \& Stocke 2003). We assume a typical distance for SGRBs, $z=0.2$.

Then the model predicts the expected flux at the observed frequency of $\nu$ as $F_{\nu} \sim 1.5 E_{50}^{4 / 3} n_{-3}^{1 / 2} \nu_{15}^{-2 / 3}\left(t / 10^{3} \mathrm{~s}\right)^{-1} \mu \mathrm{Jy}$, where $E_{50} \equiv E_{\text {iso }} /\left(10^{50} \mathrm{erg}\right), n_{-3} \equiv n /\left(10^{-3} \mathrm{~cm}^{-3}\right)$ and $\nu_{15} \equiv$ $\nu /\left(10^{15} \mathrm{~Hz}\right)$. In the X-ray band $(1 \mathrm{keV})$, the typical flux is then $\nu F_{\nu} \sim 9.3 \times 10^{-14} \mathrm{erg} \mathrm{cm}^{-2} \mathrm{~s}^{-1}$ at $t=10^{3} \mathrm{~s}$, which can be detected by existing X-ray satellites (e.g., Gehrels et al. 2005). Swift XRT can locate afterglows with an accuracy of a few arcsec, and this is reasonably accurate to discuss the association of an afterglow with a galaxy at $z \sim 0.2$. In the optical $(R)$ band, this flux corresponds to $\sim 26 \mathrm{mag}(\mathrm{AB})$ at $t=$ $10^{4} \mathrm{~s}$. Most afterglows with this level of brightness have been missed in the past and current GRB follow up observations. However, afterglows of brightest SGRBs $\left(E_{\text {iso }} \sim 10^{51}\right)$ may be detectable.

On the other hand, SGRBs ejected far from their host galaxies in the normal field would occur in much lower-density environment. The typical density in general intergalactic medium would be estimated as $n \sim \rho_{c} \Omega_{b} / m_{p} \sim 10^{-7} \mathrm{~cm}^{-3}$, where $\rho_{c}$ and $m_{p}$ are the critical density of the universe and the proton mass, respectively. The expected afterglow flux of an intergalactic SGRB is then more than one order of magnitude fainter than those of the faintest SGRB afterglows ever observed, such as GRB 050509B (Gehrels et al. 2005) and GRB 050911 (Page et al. 2006, upper limit only). Therefore it seems difficult to detect an afterglow of such an event.

\subsection{Comparison with Observations}

Berger et al. (2007) examined all 16 SGRBs that were followed up by X-ray observations with XRT of Swift or Chan$d r a$, and found that three SGRBs are likely to be associated with galaxy clusters, suggesting that the fraction of SGRBs in galaxy clusters is about $20 \%$. Considering the statistical uncertainty, it is consistent with the fraction of all stellar mass in the universe bound in galaxy clusters $(\sim 10 \%$, Fukugita, Hogen, \& Peebles 1998).

Among the three SGRBs in galaxy clusters discussed in Berger et al. (2007), GRB 050509B is apparently associated with the likely host galaxy at the cluster redshift of $z=0.226$. The offset of GRB 050509B from its host is 21$56 \mathrm{kpc}$, corresponding to $6-16 r_{\text {half }}$. The galaxy cluster that contains the host is composed of two subclusters, and the host galaxy is located at the center of the minor subcluster that is about $270 \mathrm{kpc}$ away from the center of the major subcluster (Gehrels et al. 2005; Bloom et al. 2006). The location of GRB 050911 is in a cluster of $z=0.165$, but its afterglow was too faint to associate it with any particular galaxy (Page et al. 2006). GRB 050813 has three candidate host galaxies near its Swift XRT location, and the galaxies belong to two different galaxy clusters at $z=0.72$ and $z=1.8$ (Berger 2005, 2006). Clearly, the current sample is too small to derive any implications from a comparison with our results. Future satellites for GRB study might detect SGRBs more efficiently leading to a much larger sample of SGRBs.

Observations of intracluster diffuse light, stars, type Ia supernovae, and planetary nebulae indicate that some stars in a galaxy cluster are in intracluster medium, perhaps removed from member galaxies (Vílchez-Gómez et al. 1994; Okamura et al. 2002; Durrell et al. 2002; Gal-Yam et al. 2003; Gerhard et al. 2007). The fraction of intracluster stars in all stars in a cluster is uncertain, but observational estimates are typically $\sim 5-20 \%$. These fractions are consistent with our estimate of stars outside the tidal radius (see \$2.3). On the other hand, $f_{\text {esc }}$ of SGRBs could be much higher than these, up to $\sim 80 \%$ depending on the model parameters. If such a 
higher fraction of intracluster SGRBs than that of intracluster stars is observed in the future, it would indicate the effect of kick velocities on compact binaries, giving a further support for the compact binary hypothesis of SGRBs. Note that this test is difficult in the intergalactic field, since we do not know the fraction of intergalactic stars and afterglows of intergalactic SGRBs are difficult to detect.

\section{CONCLUSIONS}

We investigated the escape of compact binaries from their host galaxies in galaxy clusters, which is enhanced by the tidal force of the cluster gravity compared with general fields. We found that the escape probability heavily depends on the uncertain distribution of subhalos associated with member galaxies. If the DM substructure has been destroyed by interactions in a galaxy cluster and the escape of a binary is determined mainly by gravity of stellar mass, most of compact binaries in galaxy clusters should escape and become hostless intracluster SGRBs. On the other hand, if the DM subhalos are associated to member galaxies with a similar amount to field galaxies, the enhancement of escape fraction is only modest compared with field galaxies: about $20 \%$ for clusters while $\sim 10 \%$ for field galaxies.

Though the current observed data set is not sufficient to be compared quantitatively with our results, statistics of SGRB association with cluster galaxies in the future data will give us important information for the dark matter distribution in clusters, intracluster stars in clusters, as well as the origin of SGRBs.

We would like to thank an anonymous referee for useful comments. This work was supported by the Grant-in-Aid for the 21st Century COE "Center for Diversity and Universality in Physics" from the Ministry of Education, Culture, Sports, Science and Technology (MEXT) of Japan.

\section{REFERENCES}

Berger, E. 2005, GRB Coordinates Network, 3801

Berger, E. 2006, preprint (astro-ph/ 0602004)

Berger, E., et al. 2005, Nature, 438, 988

Berger, E., Shin, M.-S., Mulchaey, J. S., \& Jeltema, T. E. 2007, ApJ, 660, 496

Blanton, M. R., et al. 2001, AJ, 121, 2358

Bloom, J. S., Sigurdsson, S., \& Pols, O. R. 1999, MNRAS, 305, 763

Bloom, J. S., et al. 2007, ApJ, 654, 878

Bloom, J. S., et al. 2006, ApJ, 638, 354

Bulik, T., Belczyński, K., \& Zbijewski, W. 1999, MNRAS, 309, 629

Bullock, J. S., Kolatt, T. S., Sigad, Y., Somerville, R. S., Kravtsov, A. V.,

Klypin, A. A., Primack, J. R., \& Dekel, A. 2001, MNRAS, 321, 559

de Vaucouleurs, G., \& Olson, D. W. 1982, ApJ, 256, 346

Durrell, P. R., Ciardullo, R., Feldmeier, J. J., Jacoby, G. H., \& Sigurdsson, S. 2002, ApJ, 570, 119

Fox, D. B., et al. 2005, Nature, 437, 845

Fryer, C. L., Woosley, S. E., \& Hartmann, D. H. 1999, ApJ, 526, 152

Fukugita, M., Hogan, C. J., \& Peebles, P. J. E. 1998, ApJ, 503, 518

Gal-Yam, A., Maoz, D., Guhathakurta, P., \& Filippenko, A. V. 2003, AJ, 125,1087

Gehrels, N., et al. 2005, Nature, 437, 851

Gerhard, O., Arnaboldi, M., Freeman, K. C., Okamura, S., Kashikawa, N., \& Yasuda, N. 2007, preprint (astro-ph/0703770)

Hansen, S. M., Timothy, A. M., Risa, H. W., \& James, A. 2005, ApJ, 633, 122

Hernquist, L. 1990, ApJ, 356, 359

Hobbs, G., Lorimer, D. R., Lyne, A. G., \& Kramer, M. 2005, MNRAS, 360, 974

Lacey, C., \& Cole, S. 1993, MNRAS, 262, 627
Lee, W. H., Ramirez-Ruiz, E., \& Granot, J. 2005, ApJ, 630, L165

Lee, W. H., \& Ramirez-Ruiz, E. 2007, zNew J. Phys., 9, 17

Lewis, A. D., Buote, D. A., \& Stocke, J. T. 2003, ApJ, 586, 135

Murante, G., Giovalli, M., Gerhard, O., Arnaboldi, M., Borgani, S., \& Dolag, K. 2007, MNRAS, 377, 2

Nakar, E. 2007, Phys. Rep., 442, 166

Navarro, J. F., Frank, C. S., \& White, S. D. 1995, MNRAS, 275, 720

Oemler, A. 1974, ApJ, 194, 1

Okamura, S., et al. 2002, PASJ, 54, 883

Page, K. L., et al. 2006, ApJ, 637, L13

Panaitescu, A., \& Kumar, P. 2001, ApJ, 560, L49

Piran, T. 2004, preprint (astro-ph/ 0405503)

Ransom, S. M., Kaspi, V. M., Ramachandran, R., Demorest, P., Backer, D.

C., Pfahl, E. D. Ghigo, F. D., \& Kaplan, D. L. 2004, ApJ, 609, L71

Sari, R., Piran, T., \& Narayan, R. 1998, ApJ, 497, L17

Schmidt, R. W., \& Allen, S. W. 2006,preprint (astro-ph/0610038)

Shin, M. -S., \& Berger, E. 2007, ApJ, 660, 1146

Soderberg, A. M., et al. 2006, ApJ, 650, 261

Spergel, D. N., et al. 2003, ApJS, 148, 175

Thomas, T., \& Katgerd, P. 2006, A\&A, 446, 31

Totani, T., \& Yoshii, Y. 2000, ApJ, 540, 81

Tutukov, A. V., \& Yungelson, L. R. 1994, MNRAS, 268, 871

Vílchez-Gómez, R., Pello, R., \& Sanahuja, B. 1994, A\&A, 283, 37

Villasenor, J. S., et al. 2005, Nature, 437, 855

Wex, N., Kalogera, V., \& Kramer, M. 2000, ApJ, 528, 401

Willems, B., Kalogera, V., \& Henninger, M. 2004, ApJ, 616, 414

Yamada, T., et al. 2005, ApJ, 634, 861 
TABLE 1

ESCAPE FRACTIONS IN GALAXY CLUSTER $f_{\text {esc }}\left(<R_{\text {vir }}\right)$ AND IN FIELD

\begin{tabular}{crrr}
\hline$\sigma_{k}(\mathrm{~km} / \mathrm{s})$ & Cluster (i) & Cluster (ii) & Field \\
\hline 300 & 0.79 & 0.20 & 0.10 \\
100 & 0.69 & 0.16 & 0.09 \\
\hline
\end{tabular}

NotE. - The columns labeled as Cluster (i) and (ii) give the escape fractions corresponding to (i) the no DM subhalo case and (ii) the preserved DM subhalo case discussed in $\$ 2.1$ 
Niino \& Totani

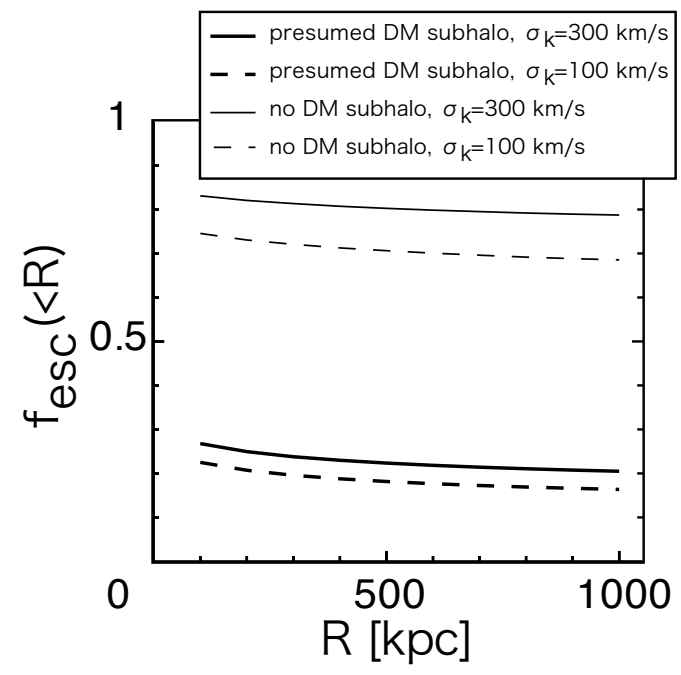

FIG. 1. - The escape fraction of compact binaries from host galaxies in a galaxy cluster averaged within $R$, where $R$ is the distance from the center of the galaxy cluster. (The virial radius of the cluster is $1 \mathrm{Mpc}$.) The models with no dark matter subhalos is shown by thin lines, while the models with preserved subhalos are shown by thick lines. The solid and dashed lines are for different values of the standard deviation of the kick velocity distribution, $\sigma_{k}=300$ and $100 \mathrm{~km} / \mathrm{s}$, respectively. 